\section{Le rôle du noyau du tractus solitaire dans la détection et l'intégration de multiples signaux métaboliques}

Clémence Blouet
Albert Einstein College of Medicine, Diabetes Research Center, 1300 Morris Park avenue, Golding 501, Bronx, 10461, New York, États-Unis.

clemence.blouet@einstein.yu.edu
> L'obésité représente un enjeu majeur de santé publique. La prévalence de l'obésité atteint désormais des proportions épidémiques, avec près de $10 \%$ de la population mondiale présentant un indice de masse corporelle supérieur à 30 (critère de définition de l'obésité). La physiopathologie de l'obésité est complexe et fait intervenir des facteurs génétiques, environnementaux et culturels dont l'interaction favorise un déséquilibre positif de la balance énergétique. Ce déséquilibre est associé à l'apparition précoce d'un excès chronique de substrats énergétiques dans l'organisme. Ce dernier a des conséquences délétères sur la fonction de multiples types cellulaires, et il est responsable de l'apparition du surpoids ainsi que de nombreuses comorbidités liées à l'obésité, telles le diabète de type 2, les maladies cardiovasculaires et le cancer. Pour prévenir cet excès de substrats énergétiques, l'organisme dispose de mécanismes de détection de l'énergie disponible couplés à la coordination d'effecteurs qui déterminent la prise alimentaire et l'utilisation énergétique. L'identification et la caractérisation de ces mécanismes sont donc essentielles à la compréhension de l'étiologie précoce de l'obésité et des dysfonctionnements métaboliques associés.

\section{Le cerveau : maillon central} de la régulation de l’homéostasie énergétique

L'hypothèse de l'importance du système nerveux central dans la régulation de la balance énergétique a été envisagée dès le milieu de XIX ${ }^{e}$ siècle par le physiologiste Claude Bernard et plusieurs de ses contemporains. Mais c'est seulement avec les expériences de lésions électrolytiques de A.W. Hetherington et S.W. Ranson un siècle plus tard que le rôle du cerveau, et plus précisément de l'hypothalamus médiobasal, dans la régulation de la prise alimentaire et la balance énergétique a été directement formulé [1]. Les découvertes récentes, tirant parti du développement de nouvelles techniques de biologie moléculaire, ont confirmé les conclusions de ces expériences avec une plus grande résolution neuroanatomique et neurochimique.

Le modèle actuel positionne le système nerveux central en chef d'orchestre de la régulation de la balance énergétique. Certaines zones du cerveau sont spécialisées dans la détection du statut énergétique de l'organisme, en partie grâce à un bagage de récepteurs et d'enzymes spécifiques. Elles représentent une zone unique de convergence neuroanatomique de multiples signaux relatifs à la disponibilité de l'énergie dans l'organisme (Figure 1). Elles reçoivent les projections vagales venant du tractus digestif et véhiculant les informations postingestives nécessaires à la mise en place du rétrocontrôle négatif régulant la taille des repas [2]. Elles disposent de récepteurs hormonaux leur permettant de détecter les signaux de disponibilité aiguë (GLPl [glucagonlike peptide 1], CCK [cholécystokinine], ghréline) ou chronique (leptine, insuline) de l'énergie dans l'organisme [3]. Elles détectent également directement les niveaux circulants de glucose, d'acides gras et d'acides aminés [4, 5]. L'intégration de ces divers signaux produit un ensemble de rétrocontrôles négatifs coordonnés régulant de multiples déterminants comportementaux et métaboliques de l'homéostasie énergétique.

Les noyaux cérébraux sensibles aux signaux de disponibilité énergétique sont particulièrement concentrés au niveau de l'hypothalamus. Ainsi, l'hypothalamus médiobasal (noyaux ventromédian et arqué de l'hypothalamus) est historiquement considéré comme la pierre d'angle de la détection et de l'intégration de l'ensemble de ces signaux (Figure 1). Les divers signaux de disponibilité énergétique de l'organisme modifient l'activité électrique et synaptique de ces neurones spécialisés dans la détection métabolique. Ces signaux engagent une série complexe de processus neurochimiques et électrophysiologiques qui aboutissent à l'activation de zones du tronc cérébral qui régulent directement les effecteurs de l'homéostasie énergétique (prise alimentaire, dépense énergétique, flux métaboliques hépatiques, métabolisme du tissu adipeux) via l'innervation sympathique et parasympathique des multiples tissus périphériques. Ce modèle représente la 


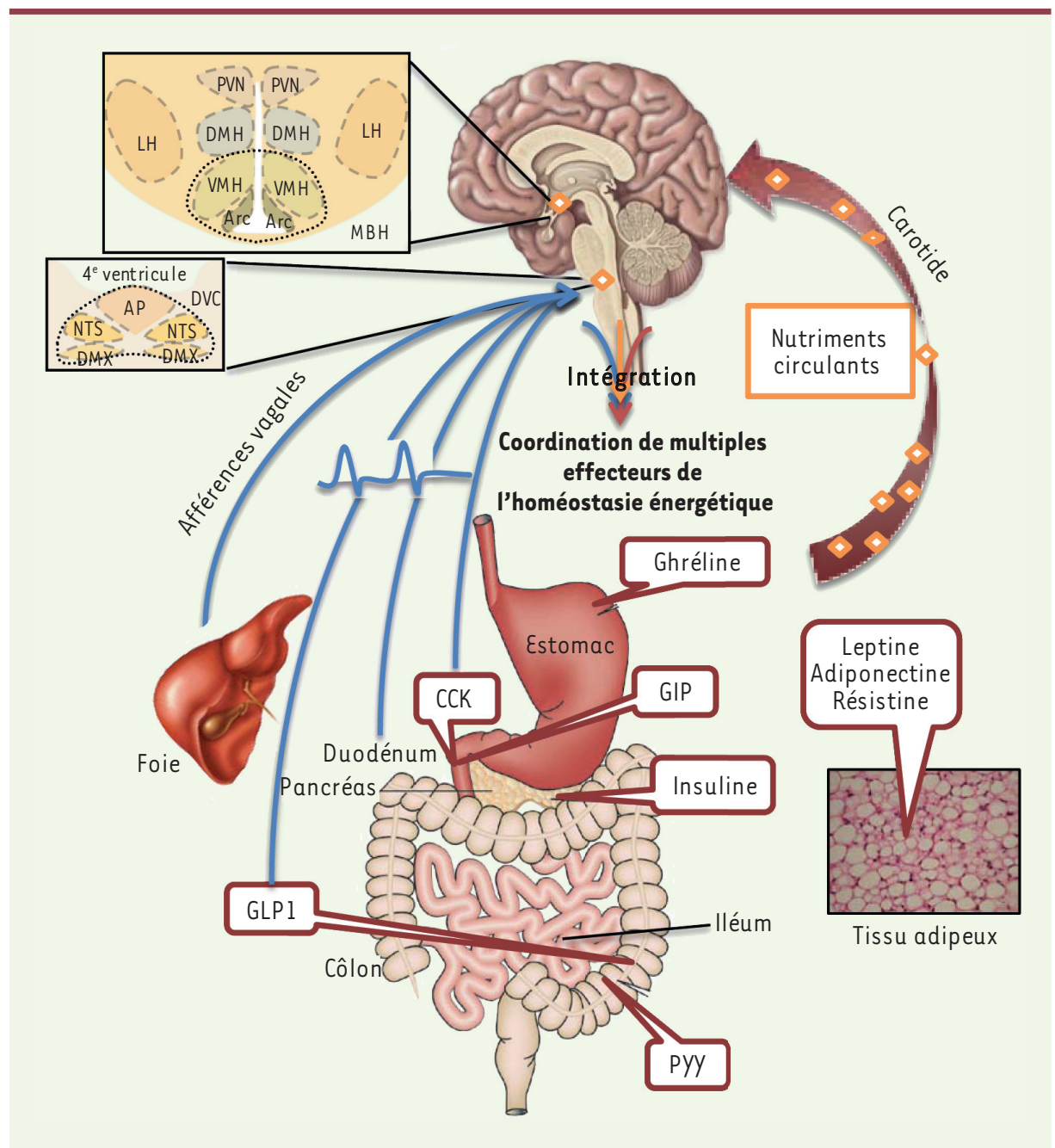

Figure 1. Convergence de multiples signaux du statut énergétique de l'organisme dans le système nerveux central. Certains neurones spécialisés du système nerveux central, les neurones senseurs métaboliques, reçoivent des afférences neurales provenant du tractus digestif véhiculant des informations relatives aux repas ingérés, et détectent les signaux humoraux de disponibilité aiguë et chronique de l'énergie dans l'organisme, ainsi que les nutriments circulants. Cette information est intégrée pour produire un rétrocontrôle coordonné régulant de multiples déterminants métaboliques et comportementaux de l'homéostasie énergétique. Arc : noyau de l'hypothalamus arqué ; DMH : noyau de l'hypothalamus dorsomédial ; LH : noyau de I'hypothalamus latéral; MBH : noyau de l'hypothalamus médiobasal; PVN : noyau paraventriculaire; VMH : noyau de l'hypothalamus ventromédian; DVC : noyaux du tronc cérébral formant le complexe dorsovagal ; AP : aire postrema ; DMX : noyau X du nerf vague ; NTS : noyau du tractus solitaire ; CCK : cholécystokinine ; GIP : polypeptide gastrique inhibiteur ; GLPl : glucagon-like peptide 1 ; PYY : peptide Yy.

vision traditionnelle de la détection des signaux métaboliques par le système nerveux central. Cependant, un ensemble de travaux récents remettent en cause cet aspect « centralisateur» de l'hypothalamus et offrent les premières preuves expérimentales en faveur d'un aspect intégratif de ce système.
Détection des signaux énergétiques : une fonction assurée par un réseau de noyaux cérébraux

L'hypothèse selon laquelle les neurones senseurs métaboliques ne sont pas anatomiquement limités à I'hypothalamus, mais au contraire distribués au sein du système nerveux central, a émergé avec les travaux d'Harvey

Grill et al. Ces travaux reposent notamment sur l'utilisation du modèle du rat décérébré dont les parties antérieures (contenant l'hypothalamus) et postérieures du cerveau sont dissociées de manière chirurgicale [6]. Le rat décérébré détecte les signaux de satiété postingestifs et régule la taille de ses repas de la même manière que le rat intact, malgré la destruction des communications neurales entre le système gastro-intestinal et l'hypothalamus. Ces travaux permettent de conclure que le cerveau postérieur est suffisant pour l'intégration des signaux métaboliques postingestifs dans la régulation de la prise alimentaire, et ils remettent en question le dogme selon lequel le rôle du NTS (nucleus of the solitary tract) se limite à celui d'un relais neural vers l'hypothalamus.

Par ailleurs, l'expression des récepteurs hormonaux nécessaires à la détection de diverses hormones métaboliques (leptine, insuline, incrétines) dans des noyaux hypothalamiques distincts de l'hypothalamus ventromédian, ainsi que dans plusieurs zones extra-hypothalamiques, notamment le NTS dans le tronc cérébral caudomédian ( $\mathrm{cmNTS}$ ), suggère que des groupes de neurones anatomiquement distribués au sein du système nerveux central pourraient contribuer à la détection du statut métabolique de l'organisme [7]. L'utilisation de l'électrophysiologie sur coupes de cerveau provenant de ces diverses zones cérébrales a confirmé que l'activation de ces récepteurs est bien couplée à une modification de l'activité électrique neuronale. Des données pharmacologiques - analysant les conséquences compor- 


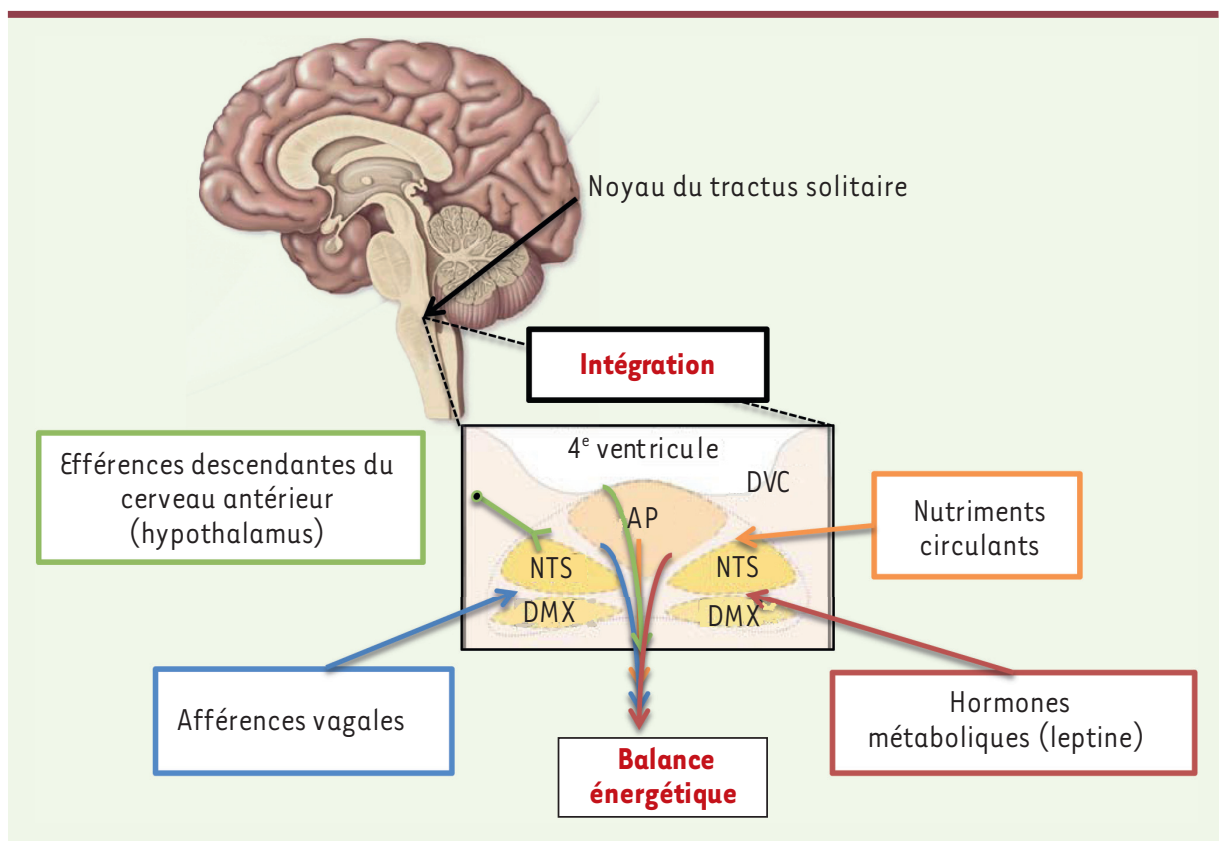

Figure 2. Le noyau du tractus solitaire : point de convergence et d'intégration de multiples signaux énergétiques.

tementales et métaboliques, chez le rat vigile, de l'activation locale de ces récepteurs dans divers noyaux cérébraux - indiquent que l'action de ces hormones métaboliques sur des neurones localisés à l'extérieur de l'hypothalamus médiobasal contribue au contrôle de la balance énergétique [7]. Ces résultats ont été confirmés dans la plupart des cas par des approches invalidant de manière restreinte au niveau neuroanatomique ou neurochimique (par siARN ou knockout) l'expression des récepteurs hormonaux ou des protéines impliquées dans les voies de signalisation qui leur sont associées [8]. Ainsi, le rôle de divers noyaux hypothalamiques, notamment l'hypothalamus latéral, dorsomédian, paraventriculaire, et les noyaux extra-hypothalamiques dans le cerveau postérieur (cmNTS) et dans le cerveau moyen (aire tegmentale ventrale), a été récemment mis en évidence.

Les mêmes approches ont été utilisées pour l'étude des neurones senseurs de nutriments, mais celle-ci est compliquée par l'absence de marqueurs cellulaires (récepteur, transporteur, enzyme ou neurotransmetteur) exprimés uni- quement par ces neurones et qui permettraient de les identifier. L'électrophysiologie sur coupes et les injections cérébrales de nutriments ont principalement été appliquées à la caractérisation de neurones sensibles au nutriments dans l'hypothalamus médiobasal [9-11], mais des neurones sensibles au glucose ont également été identifiés dans plusieurs autres régions hypothalamiques et extra-hypothalamiques [12]. Récemment, la détection des acides aminées par des neurones du cmNTS et son importance dans la régulation de la balance énergétique ont été mises en évidence [13], suggérant que la détection centrale des acides aminés dans la régulation de l'homéostasie énergétique est également assurée par plusieurs noyaux cérébraux.

Si au sein du système nerveux central la fonction de détection des signaux de disponibilité énergétique n'est pas restreinte à l'hypothalamus médiobasal, ce dernier est toujours considéré à l'heure actuelle comme le principal centre de convergence de l'ensemble des signaux métaboliques (afférences périphériques, hormones et nutriments). Cependant, le
cmNTS s'apparente également à un centre de convergence de ces signaux, et plusieurs études corroborent l'aspect intégratif de la détection métabolique au sein du cmNTS.

Le noyau du tractus solitaire, un senseur du statut énergétique de l'organisme Une série de découvertes récentes positionnent le $\mathrm{cmNTS}$ comme un centre de convergence des signaux métaboliques, au même titre que I'hypothalamus médiobasal [14] (Figure 2). Le cmNTS a été initialement considéré comme le site de terminaison des afférences vagales recevant les signaux postingestifs nécessaires à la mise en place du rétrocontrôle négatif régulant la taille des repas [2]. Cependant, ce noyau détecte également les signaux hormonaux du statut énergétique de l'organisme, ainsi que les taux de nutriments circulants $[13,14]$. Les capacités intégratives du cmNTS ont été initialement suggérées par des données d'électrophysiologie recueillies lors de l'analyse de l'interaction de différents signaux intestinaux de nature mécanique et chimique [15]. Celles-ci ont ensuite été étendues à l'intégration des signaux intestinaux avec les afférences mélanocortinergiques descendant de l'hypothalamus, et les signaux hormonaux de disponibilité énergétique. Ainsi, l'administration dans le cmNTS de leptine ou de melanotan 2 (un agoniste des récepteurs 3 et 4 aux mélanocortines) à des doses sans effet sur la prise alimentaire, augmente la réponse anorexique qu'induit une administration intrapéritonéale de cholécystokinine (CCK) [16, 17]. Plus récemment, nous avons montré, dans des expériences similaires, que la détection de la leucine par les neurones du cmNTS est également intégrée aux signaux neuronaux et hormonaux 
du statut énergétique de l'organisme [13]. Ces signaux activent des voies de signalisation intracellulaires communes, et notamment la voie $\varepsilon r k l / 2$ (extracellular signal-regulated kinase 1/2), proposée comme un intégrateur moléculaire des signaux métaboliques dans le cmNTS $[13,16,18]$. $\diamond$

Role of the nucleus of the solitary tract in the detection and integration of multiple metabolic signals

\section{LIENS D'INTÉRÊT}

L'auteur déclare n'avoir aucun lien d'intérêt concernant les données publiées dans cet article.

\section{RÉFÉRENCES}

1. Hetherington AW, Ranson SW. Hypothalamic lesions and adiposity in the rat. Anat Rec $1940 ; 78: 149-72$.

2. Moran TH, Ladenheim $\varepsilon \varepsilon$, Schwartz GJ. Within-meal gut feedback signaling. Int J Obes Relat Metab Disord $2001 ; 25$ : S39-41.
3. Woods SC, D’Alessio DA. Central control of body weight and appetite. J Clin Endocrinol Metab 2008 ; 93 : S3750.

4. Jordan SD, Konner AC, Bruning JC. Sensing the fuels: glucose and lipid signaling in the CNS controlling energy homeostasis. Cell Mol Life Sci 2010 ; 67 : 325573.

5. Schwartz GJ. Central leucine sensing in the control of energy homeostasis. Endocrinol Metab Clin North Am $2013 ; 42: 81-7$.

6. Grill HJ, Kaplan JM. Interoceptive and integrative contributions of forebrain and brainstem to energy balance control. Int J Obes Relat Metab Disord 2001 ; $25:$ : $73-7$.

7. Grill HJ. Distributed neural control of energy balance: contributions from hindbrain and hypothalamus. Obesity 2006 ; 14 : S216-21.

8. Yeo GS, Heisler LK. Unraveling the brain regulation of appetite: lessons from genetics. Nat Neurosci 2012 ; $15: 1343-9$.

9. Le Foll C, Irani BG, Magnan C, et al. Characteristics and mechanisms of hypothalamic neuronal fatty acid sensing. Am J Physiol Regul Integr Comp Physiol 2009; $297:$ R655-64.

10. Jo YH, Su Y, Gutierrez-Juarez R, Chua S Jr. Oleic acid directly regulates POMC neuron excitability in the hypothalamus. J Neurophysiol 2009 ; 101 : 2305-16.

11. Blouet C, Jo YH, Li X, Schwartz GJ. Mediobasal hypothalamic leucine sensing regulates food intake through activation of a hypothalamus-brainstem circuit. J Neurosci 2009 ; 29 : 8302-11.

12. Thorens B. Sensing of glucose in the brain. Handb Exp Pharmacol $2012: 277-94$

13. Blouet C, Schwartz GJ. Brainstem nutrient sensing in the nucleus of the solitary tract inhibits feeding. Cell Metab $2012 ; 16$ : 579-87.

14. Grill HJ, Hayes MR. Hindbrain neurons as an essential hub in the neuroanatomically distributed control of energy balance. Cell Metab 2012 ; 16 : 296-309.

15. Schwartz GJ, Moran TH. Sub-diaphragmatic vagal afferent integration of meal-related gastrointestinal signals. Neurosci Biobehav Rev 1996 ; $20: 47-56$.

16. Berthoud HR, Sutton GM, Townsend RL, et al. Brainstem mechanisms integrating gut-derived satiety signals and descending forebrain information in the control of meal size. Physiol Behav 2006 ; 89 : 517-24.

17. Williams DL, Baskin DG, Schwartz MW. Hindbrain leptin receptor stimulation enhances the anorexic response to cholecystokinin. Am J Physiol Regul Integr Comp Physiol 2009 ; 297 : R1238-46.

18. Hayes MR, Bradley L, Grill HJ. Endogenous hindbrain glucagon-like peptide-l receptor activation contributes to the control of food intake by mediating gastric satiation signaling. Endocrinology 2009; 150 : 2654-9.

\section{NOUVELLE}

\section{Vers un ribosome artificiel \\ Une molécule pour fabriquer des molécules}

Guillaume De Bo, David A. Leigh
University of Manchester, school of chemistry, Oxford road, M13 9PL, Manchester, Royaume Uni. guillaume.debo@manchester.ac.uk

Parmi toutes ces machines, la plus spectaculaire reste sans doute le ribosome. lule. De fait, chaque cellule vivante est constituée d'une myriade de machines moléculaires effectuant les tâches les plus diverses. Par exemple, les protéines moteurs, telles que les dynéines et les kinésines, utilisent l'énergie contenue dans l'adénosine triphosphate (ATP) pour effectuer le transport de vésicules en «marchant» le long des microtubules; les moteurs moléculaires utilisent l'énergie d'un gradient de protons pour assurer le battement des flagelles ou la synthèse d'ATP; enfin, les hélicases et les topoisomérases agissent sur I'ADN, pour, respectivement, en séparer les brins et en modifier la topologie [5].

\section{Conception et caractéristiques d'un ribosome artificiel}

Le ribosome, que l'on trouve dans chaque cellule vivante, est un énorme complexe ribonucléoprotéique qui assure la synthèse des protéines au départ de l'information contenue dans I'ARN messager [6]. Lorsque le ribosome entre en action, les deux sous-unités qui le composent viennent s'amarrer l'une à l'autre en enserrant la chaîne d'ARN en leur sein, tel un poing serrant une corde. Une fois fixé, le ribosome coulisse le long de l'ARN pour assembler les acides 